\title{
Palletizing robot control system based on Trio motion controller
}

\author{
Gui-Zhi ZHAO ${ }^{1, a}$, \\ ${ }^{1}$ Inner Mongolia University for the Nationalities, College of Mechanical Engineeringe, TongLiao, \\ 028000, China; \\ aguizhi_84@yahoo.com.cn
}

Keywords: Palletizing robot, Trio motion controller, Six degree of freedom robot, Openness, Control system

\begin{abstract}
Improvement of the level of industrialization accelerates the development of logistics automation technology. The application of palletizing robot is becoming more and more, its flexibility is improving continuously. In this paper, the palletizing robot control system is designed based on trio motion controller and palletizing robot uses six degree of freedom structure, hardware and software are designed. The system uses modular design to improve the system openness and portability, and it greatly improves the efficiency of software development. Double CPU PC makes real-time and non real-time program operate separately, it can improve the efficiency of the system.
\end{abstract}

\section{Introduction}

With the continuous development of industrial automation, robot is used more and more widely in various industries [1]. Palletizing technology is a novel technology for logistics automation and palletizing technology can make the materials stacked in the stacking in accordance with the requirements, it is convenient for later handling, storage, handling, transportation, and other works [2]. Industrialized mass production also accelerates the automation of palletizing technology, palletizing technology automation can ensure material uniformity, so as to guarantee the quality of the material. It can ensure the safety of operators, reduce material waste and damage [3]. With the continuous expansion of production scale and improvement of industrial automation, palletizing robot flexibility continuously upgrades [4]. Therefore, the palletizing robot with low cost and high performance has broad market prospects. In recent years, the six degree of freedom robot because of its broad work range, flexible action and other advantages is widely used in manufacturing field [5]. A six degree of freedom palletizing robot is designed in this paper based on Trio motion controller, the system uses a modular design, and it can improve the system's flexibility and openness.

\section{Robot hardware platform design}

Overall design. In order to increase the system flexibility, and the system function is expanded, the control system must be open, controller structure must also be open. The new task requires new device matching, therefore the hard ware also needs to open [6]. The control system of the robot is designed using hierarchical and modular. As shown in Figure 1, from the functions of the system is divided into management layer, control layer, driver layer and control object. 


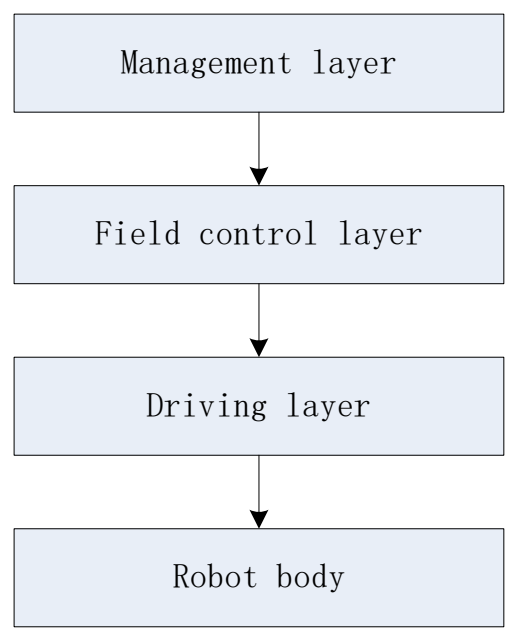

Figure1. System function

Hardware design. After the design of the overall system design, each module needs to be designed separately. The system hardware integration is shown in figure 2 . The introduction will be following.

Management is mainly used to deal with some real-time tasks, users will pass motion parameters to the field control layer through management, and it will receive field control layer feedback information on the state of the system. The design of management requires convenient operation, and it can be extended. Advantech IPC has strong anti-interference, good expansibility, so use Advantech IPC as the control layer of hardware.

Field control layer is mainly to complete the real-time tasks, and to perform the corresponding control program, and at the same time feedbacks information to management state. The field control layer adopts Trio controller as hardware. TRIO digital motion controller is a kind of digital motion controller developed based on micro processing technology of high precision and high function by Trio motion technology Ltd. TRIO digital motion controller has good expansibility and provides a variety of functional modules, it can configure according to the application requirements of on-demand portfolio and it improves the system openness and portability.

The driver layer and the control object are the servo system and the robot body respectively. Schneider motor servo system is used. The robot uses six degrees of freedom structure.

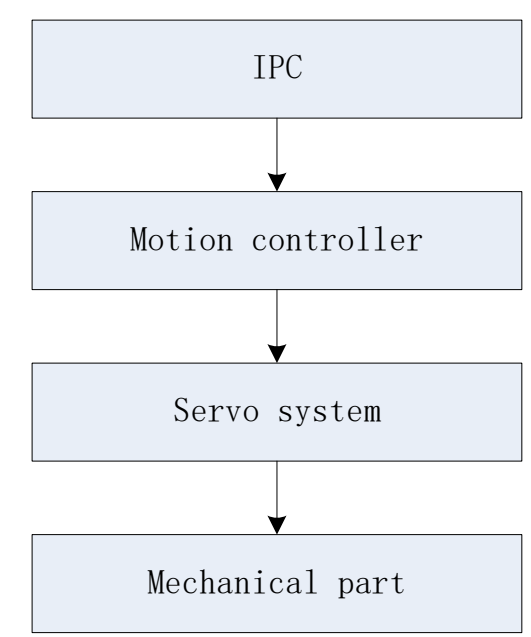

Figure2. Hardware integration

\section{Software design}

Software structure design. Robot system is mainly used to control the robot palletizing, as shown in Figure 3, according to the system hardware design, software takes the upper and lower computer 
design. The upper and lower computer software are designed. PC program is developed in computer, programs of the lower computer is written through the PC, trio motion controller provides special programming software Motion Perfect, the program can debug on it and debugging program can be directly transferred to the motion controller.

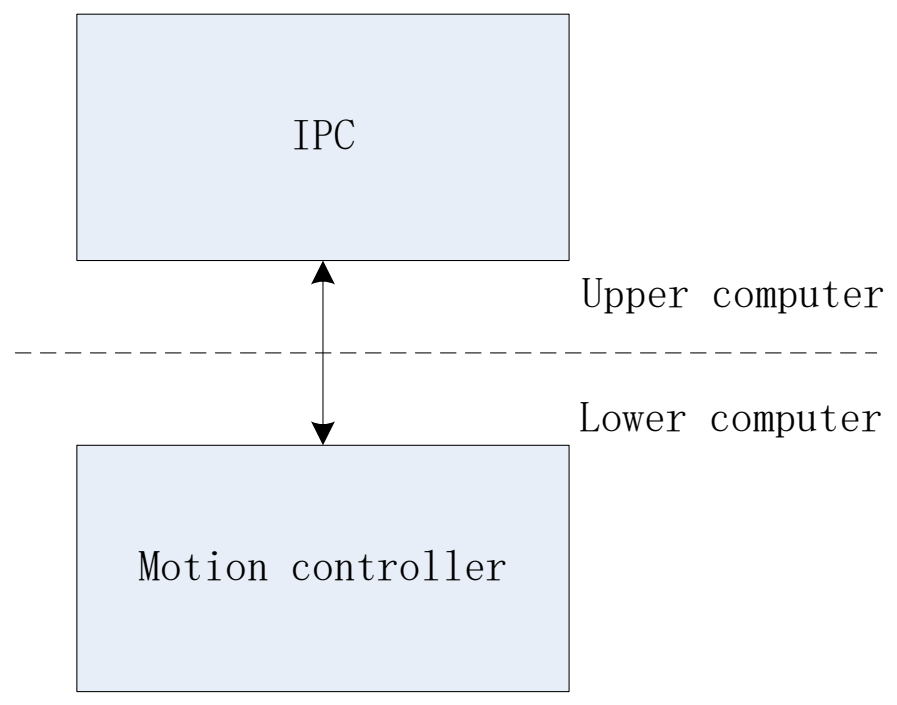

Figure3. The structure of software

Upper computer software design. PC software matches hardware management layer, as shown in Figure 4, the main function of PC software are: program management, parameter management, state management and system diagnosis. Program management is used for palletizing program writing, palletizing process design, palletizing parameter setting. Parameter management is mainly to set parameters, including the parameters of robot, servo motor parameters and coordinate parameters etc. State management is mainly used to initialize the state of the system, and to monitor each state. The system is mainly do the diagnosis and monitor system failure.

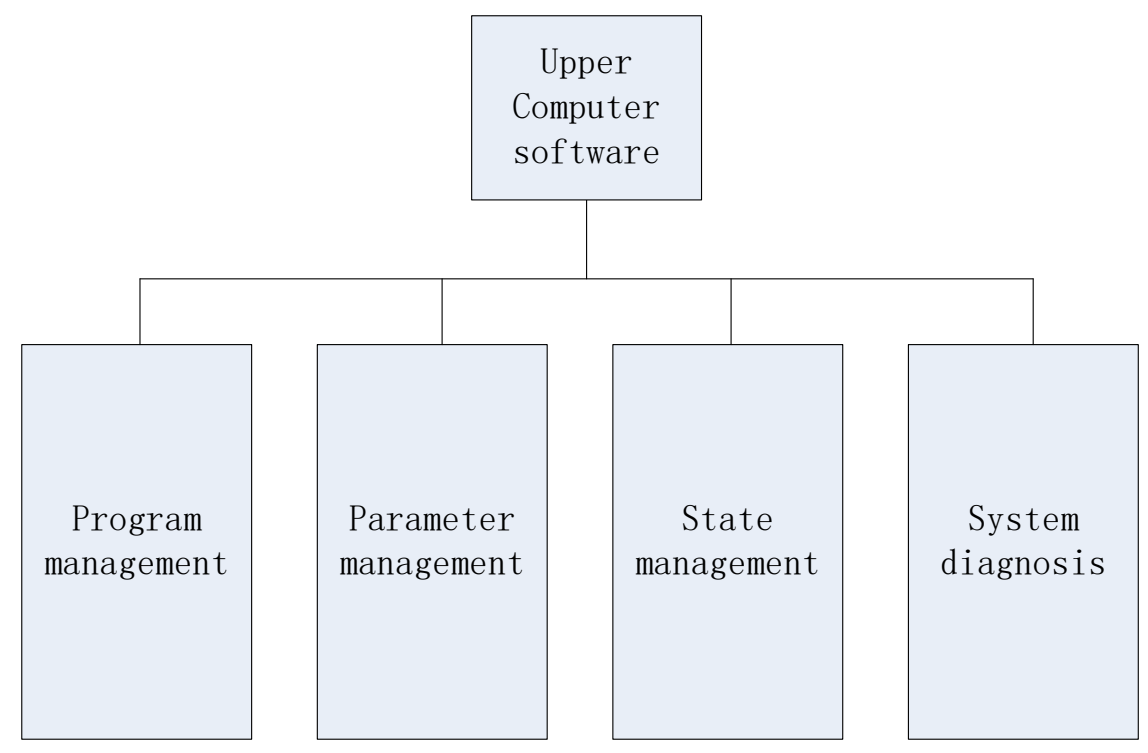

Figure4. The upper computer software

Lower computer software design. First the robot kinematics and inverse kinematics are designed, the system uses the D-H method kinematic model to determine the relationship between the motor angle and position. After the kinematic design, the completion of the software is done, the corresponding hardware is Trio motion controller. The robot working process is from capture to cycle placement. System model is shown in Figure 5, robot grabs from materials from A to point C, B is the 
point ready to place, and the cycle continues. In order to improve the efficiency of the system, the "linear+point to point" of the operation mode of palletizing is used. The working flows of the system are: the first point to point comes to point $\mathrm{A}$, then through the linear motion to $\mathrm{B}$, and then through point to point comes to point $\mathrm{C}$.

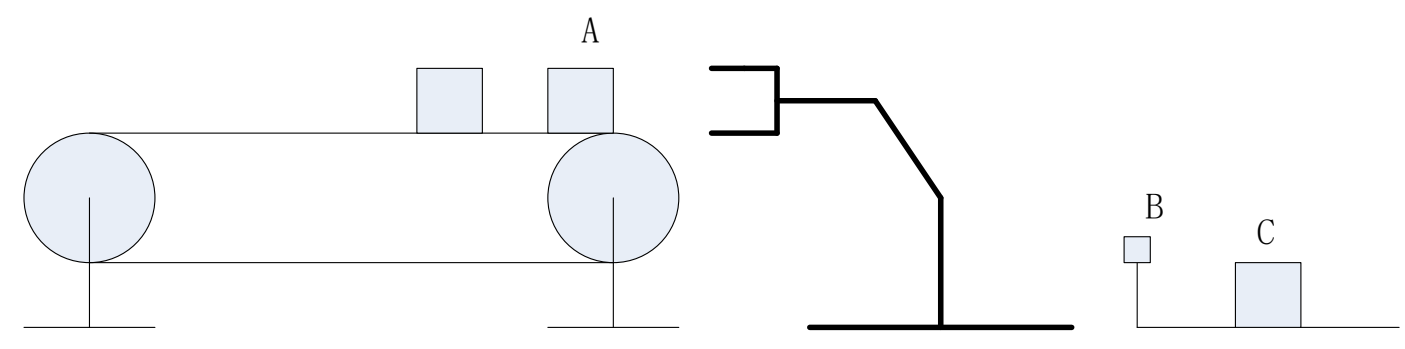

Figure5. System model

System interface design. The system needs to communicate with the position signal, indicating signal, the communication is done via the I/O interface controller. The design of I/O system is shown in table 1.

Table1. System I/O design

\begin{tabular}{lccc}
\hline & Input & & Output \\
\hline IN0 & Position signal 1 & OUT0 & Indicating signal 1 \\
IN1 & Position signal 2 & OUT1 & Indicating signal 2 \\
IN2 & Position signal 3 & OUT2 & Indicating signal 3 \\
IN3 & Position signal 4 & OUT3 & Indicating signal 4 \\
IN4 & Position signal 5 & OUT4 & Indicating signal 5 \\
IN5 & Position signal 6 & OUT5 & Indicating signal 6 \\
\hline
\end{tabular}

\section{Summary}

In this paper, according to the requirement of logistics automation technology, a six degree of freedom palletizing robot control system is designed, the system is designed based on open motion controller. The system provides a wide open, extensible platform, system hardware has compact structure, it can assembly conveniently, for further expansion. And the extension and the robot control system can connect seamlessly. The structure of the robot is reasonable, it can meet the requirements of the palletizing, production efficiency is higher, it can be widely used in material palletizing in logistics automation industry.

\section{References}

[1] Li Lei, Ye Tao, Tan Min et al. Research status and the future of mobile robot technology [J]. Robot, 2002, 24 (5): 475-480.

[2] Cao Tong, Yuan Chao, Sun Xingchu. Research and application of TV handling palletizing robot [J]. Mechanical design and manufacturing, 2002 (2): 44-46.

[3] Hu Hongguo, Gao Jianhua, Yang Ruqing. Palletizing technology overview [J]. Modular machine tool and automatic manufacturing technique, 2000, (6): 7-9.

[4] Li Chengwei, Zhu beauty, Yun Chao. Palletizing robot mechanism design and control system [J]. Mechanical and electrical engineering, 2008, 25 (12): 81-84.

[5] Han Junwei, Hong Zhou Jiang. For six degree of freedom parallel robot navigation simulation [J]. High technology letters, 2001, 11 (3): 88-90. 
[6] Zhao Chunhong, Qin Xiansheng, Tang Hong. Based on PC open CNC system [J]. Mechanical science and technology, 2005, 24 (9) 